\title{
A novel method for nanoprecision alignment in wafer bonding applications
}

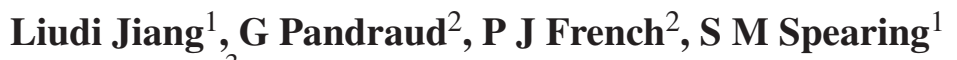 \\ and $\mathrm{M} \mathrm{Kraft}{ }^{3}$ \\ ${ }^{1}$ School of Engineering Sciences, University of Southampton, Southampton SO17 1BJ, UK \\ ${ }^{2}$ Electronic Instrumentation Laboratory, Faculty of Electrical Engineering, \\ Mathematics and Computer Science, Delft University of Technology, 2628 CD Delft, \\ The Netherlands \\ ${ }^{3}$ School of Electronics and Computer Science, University of Southampton, Southampton, \\ SO17 1BJ, UK \\ E-mail: 1.jiang@soton.ac.uk
}

Received 16 November 2006, in final form 16 January 2007

Published 26 June 2007

Online at stacks.iop.org/JMM/17/S61

\begin{abstract}
Wafer bonding has been identified as a promising technique to enable fabrication of many advanced semiconductor devices such as three-dimensional integrated circuits (3D IC) and micro/nano systems. However, with the device dimensions already in the nanometre range, the lack of approaches to achieve high precision bonding alignment has restricted many applications. With this increasing demand for wafer bonding applications, a novel mechanical passive alignment technique is described in this work aiming at nanoprecision alignment based on kinematic and elastic averaging effects. A number of cantilever-supported pyramid and V-pit microstructures have been incorporated into the outer circumference area of the to-be-bonded Si chips, respectively. The engagement between the convex pyramids and concave V-pits and the compliance of the support cantilever flexures result in micromechanical passive alignment which is followed by direct bonding between the Si chips. The subsequent infrared (IR) and scanning electron microscopy (SEM) inspections repeatedly confirmed the achievement of alignment accuracy of better than $200 \mathrm{~nm}$ at the bonding interface with good bonding quality. The impact and potential applications of the developed alignment technique are also discussed.
\end{abstract}

(Some figures in this article are in colour only in the electronic version)

\section{Introduction}

Since its inception over three decades ago, wafer bonding has been an important technique for material and microsystem integration. Many wafer bonding methods have been developed over the years enabling a wide range of semiconductor applications such as the formation of silicon on insulator (SOI) wafers $[1,2]$. In recent years, the importance of wafer bonding has also been realized in the development of multi-layered micro/nano electromechanical systems (MEMS/NEMS) [3] and three-dimensional integrated circuits (3D IC) integration $[4,5]$ aiming at creating more compact and complex systems with improved functionality. This enables semiconductor devices to be fabricated separately and bonded together at a later stage and therefore provides more freedom in design and allows more advanced semiconductor systems to be fabricated. Wafer bonding has been identified as a promising technique to enable 3D IC which features in the International Technology Roadmap for Semiconductors (ITRS). However, with line widths in CMOS manufacture already below $100 \mathrm{~nm}$ and the shrinkage of electromechanical systems into the nanometre range, the lack of effective techniques for achieving bonding alignment with submicron precision has become a critical stumbling block hindering device realization in many fields where super accurate bonding alignment is required $[6,7]$. 
Optical alignment is by far the most commonly used method to achieve bonding alignment. This is achieved by positioning one wafer with respect to another and aligning them using alignment marks observed through optical objectives. A variety of optical methods have been implemented so far; however, alignment accuracy of $1 \mu \mathrm{m}$ at best is obtained due to the large optical structural loop [8]. Although $0.5-1 \mu \mathrm{m}$ alignment has been claimed [8], complicated and expensive optical machines are required to guarantee micron-range results. Such techniques are fundamentally limited by the wavelength of light and practically limited by the mechanical positioning systems required to match the two wafers. Inspired by $\mathrm{LEGO}^{\mathrm{TM}}$ which demonstrates remarkably accurate alignment (better than $3 \mu \mathrm{m}$ ) with very low tolerances between the individual building bricks, which are fabricated by low cost injection moulding, recently a mechanical passive wafer alignment method has been reported [9] exploiting kinematic and elastic averaging. However, the alignment accuracy was obtained by optically observing the alignment marks located on the nonbonded sides of the wafer pair. This inevitably introduced optical and double-side alignment errors during the fabrication of the alignment marks, which could be well above $1 \mu \mathrm{m}$, and thus they were not able to assess the actual alignment accuracy at the bonded interface. Furthermore, wafer bonding was not achieved in this case due to the roughening of the bonding surface introduced by the fabrication processes.

In this paper, we report a practical and effective approach to achieve nanoprecision alignment accuracy for wafer bonding based on the mechanical principles of kinematic and elastic averaging. All the micromechanical structures have been designed to enable their formation using commonly available microfabrication techniques aiming towards very high bonding alignment accuracy.

\section{Design considerations}

A multitude of cantilever-supported pyramids and V-pit microstructures were designed at the outer circumference area of the Si chips $(20 \mathrm{~mm} \times 20 \mathrm{~mm})$, respectively, with a view to form kinematic couplings and elastic averaging mechanism. The centre majority area of the chips $(\geqslant 10 \mathrm{~mm} \times$ $10 \mathrm{~mm}$ ) was left blank as the bonding area. The design layout is shown in figure $1(a)$. Considering that a kinematic coupling ideally requires high precision angled surfaces, we designed pyramid and V-pit microstructures in the chips A and $\mathrm{B}$, respectively, working as kinematic coupling convex and concave features. Their smooth Si $\left\{\begin{array}{lll}1 & 1 & 1\end{array}\right\}$ sidewalls with a precise angle $\left(54.7^{\circ}\right)$ can be achieved by anisotropic $\mathrm{KOH}$ etching techniques. All the pyramid features are supported by cantilever flexures whose deflection initiates the engagement between the alignment features. The bending of the cantilevers is induced by a patterned $\mathrm{SiN}$ layer with highly compressive stress on top of the levers. Consequently, when a chip A is stacked on top of a chip B, the lowered pyramids positioned at the tips of the cantilevers are able to engage with the V-pits. The shape and position of the V-pits and pyramids provide kinematic averaging, while the compliance of the cantilever flexures introduces elastic averaging, and therefore (a)

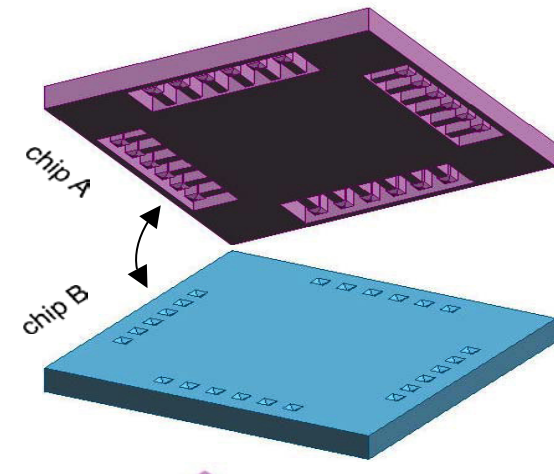

(b)

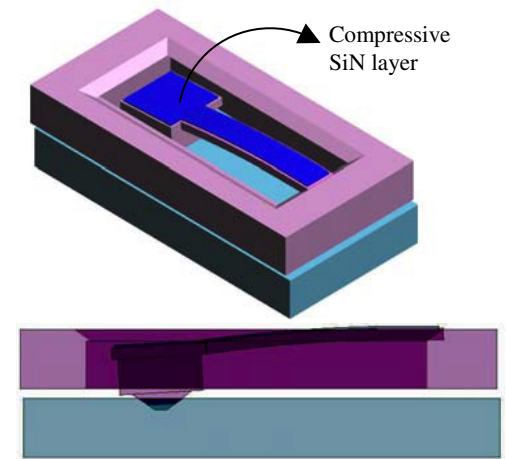

Figure 1. (a) Chip designs containing alignment features (not to scale), (b) and (c) a pair of engaged alignment features in 3D and profile view, respectively.

self-alignment is achieved as a result. The engagement concept is illustrated in figures $1(b)$ and $(c)$.

Sufficient depths for the pyramids and V-pits are required to implement the alignment concept. However, deeper etch cavities require proportionally larger design areas especially in order to achieve the required pyramid convex corner compensating structures (CCCS) associated with anisotropic $\mathrm{KOH}$ etching of Si [10]. This, in turn, limits the available bonding area. $\mathrm{KOH}$-etched patterns with larger sizes could also potentially lead to final structures with poorer dimensional tolerances. This is attributed to the bevelling effect originating from angle misalignment to the Si crystal structure during photolithography patterning [11]. In this work, compact CCCS have been designed based on a $100 \mu \mathrm{m}$ etch depth for the pyramid convex corners, using a method proposed by Zhang et al [12], which leads to the designed pyramid top and base dimensions of $356 \mu \mathrm{m}^{2}$ and $498 \mu \mathrm{m}^{2}$, respectively. Furthermore, during the alignment, it is important to position the pyramid tops initially within the open area of the V-pits guided by the chip edges so that the pyramids can slide into the V-pits and achieve engagement/alignment. Therefore, it is necessary to ensure the larger dimensions of the V-pit openings compared to the pyramid tops, consequently the V-pit dimensions were chosen to be $386 \mu \mathrm{m}^{2}$. Derived from these designed dimensions of the alignment features, a minimum cantilever displacement $d_{\min }=28 \mu \mathrm{m}$ was obtained to ensure locking is achieved between the alignment features. For a cantilever with a tip displacement $d$ and length $l$, the strain $\varepsilon$ can be calculated as

$$
\varepsilon=\frac{3 t d}{2 l^{2}}
$$




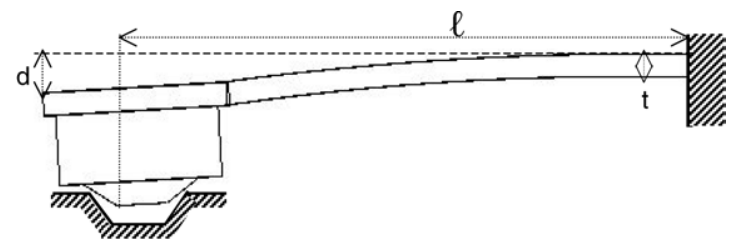

Figure 2. Schematic drawing of the designed micromechanical alignment features (not to scale).

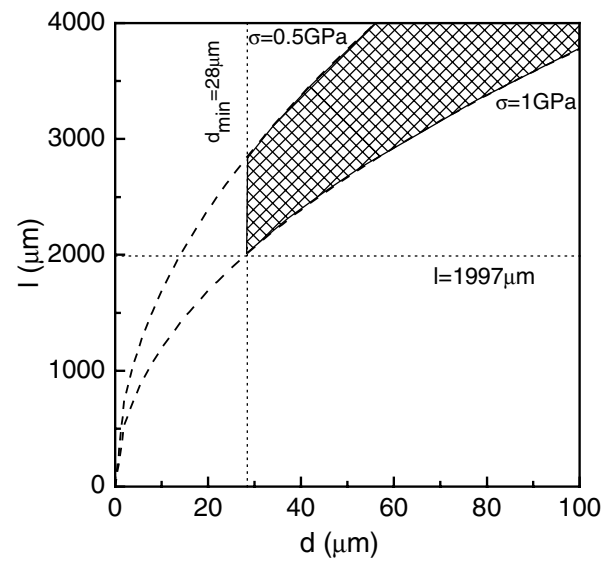

Figure 3. Cantilever length $l$ as a function of cantilever displacement $d$.

and its curvature can be expressed [13] as

$$
R \approx \frac{l^{2}}{2 d}
$$

assuming the displacement is much smaller than the overall cantilever length. The representative parameters are indicated in figure 2. Assigning the cantilever thickness $t=100 \mu \mathrm{m}$, a minimum cantilever length $l_{\min }=1450 \mu \mathrm{m}$ was attained to keep the maximum strain in the cantilevers below $0.2 \%$ in order to avoid fracture. With the stress of $0.5-1 \mathrm{GPa}$ achievable from the available $\mathrm{SiN}$ deposition facilities, the range of the cantilever lengths to be investigated can be assessed using Stoney's equation in the case of uniaxial stress [14]:

$$
\sigma=\frac{E t^{2}}{6 R t_{f}}=\frac{E t^{2} d}{3 t_{f} l^{2}}
$$

where $\sigma$ and $t_{f}$ are the stress and thickness of the SiN film and $E$ is the Young's modulus of Si. Employing $E=$ $150 \mathrm{GPa}, t=100 \mu \mathrm{m}$ and $t_{f}=3.5 \mu \mathrm{m}$, figure 3 shows the calculated length as a function of the tip displacement. The
Table 1. Designed dimensions.

\begin{tabular}{ll}
\hline Microstructures & Dimensions $(\mu \mathrm{m})$ \\
\hline Cantilever length $(l)$ & $2080-3830$ \\
Cantilever width $(w)$ & 400 \\
Cantilever thickness $(t)$ & 100 \\
Pyramid height & 100 \\
Pyramid top & 356 \\
Pyramid base & 500 \\
V-pit opening & 386 \\
Number of alignment features & 10 per pitch; 40 per chip \\
\hline
\end{tabular}

designs of the possible cantilever lengths and displacements are indicated by the shaded region. To ensure sufficient cantilever displacement under the compressive stress from the SiN film, cantilever lengths in the range of 2080-3830 $\mu \mathrm{m}$ have been determined. Chip designs with different cantilever lengths were incorporated on a 4 inch wafer with a standard thickness of approximately $500 \mu \mathrm{m}$. The designed dimensions of the key microstructures are summarized in table 1 .

Micro-vernier structures were also designed at the bonding interface for the assessment of the bonding alignment. The vernier scales were patterned and formed by $\mathrm{KOH}$ etching in the same process step as the pyramids/pits using a singlemask process. This is to ensure the precise distance between the verniers and the alignment features within each Si chip so that the vernier inspection can be used to assess the exact alignment accuracy between the pyramids and pits after aligned bonding. Figure 4 illustrates the overlapping mask designs of the vernier patterns on chips $\mathrm{A}$ and $\mathrm{B}$, respectively. There are 50 vernier lines ( $4 \mu \mathrm{m}$ wide) at each side of the middle scales and the spaces are $10 \mu \mathrm{m}$ and $9.8 \mu \mathrm{m}$ for chips A and B, respectively, which lead to $200 \mathrm{~nm}$ measurement resolution. Figure 4 shows details in the middle and end regions of the overlapping verniers, which indicate alignment exceeding the $200 \mathrm{~nm}$ resolution limit. Verniers in both $x$ and $y$ directions have been included at the corners of the chips.

\section{Fabrication}

Figure 5 shows a cross-sectional schematic illustration of the fabrication sequence for the alignment features. $(a, h) \mathrm{A}$ $100 \mathrm{~nm}$ thick SiN layer was deposited by low-pressure chemical vapour deposition (LPCVD) and patterned by reactive ion etching (RIE) with photoresist as the etch mask. $(b, i)$ A $33 \mathrm{wt} \% \mathrm{KOH}$ solution was used at $85{ }^{\circ} \mathrm{C}$ to etch $\mathrm{Si}$ anisotropically for the formation of the pyramids, V-pits and the vernier scales using the patterned $\mathrm{SiN}$ as etch masks.

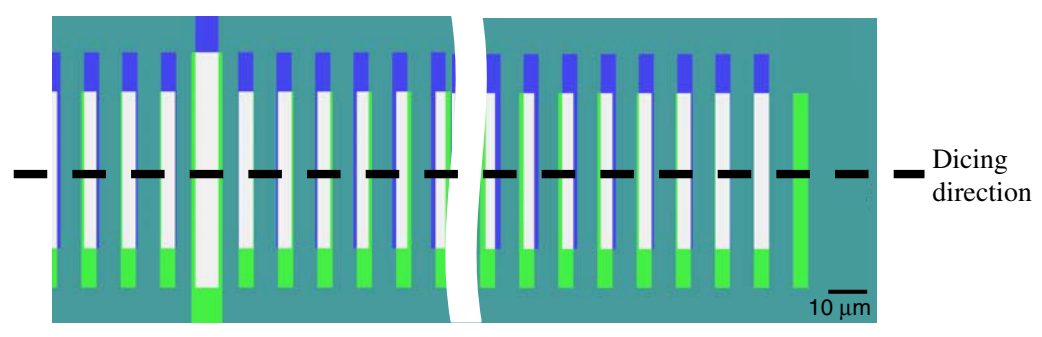

Figure 4. Overlapping mask designs of the vernier patterns in the middle and end regions representing a perfect alignment. 


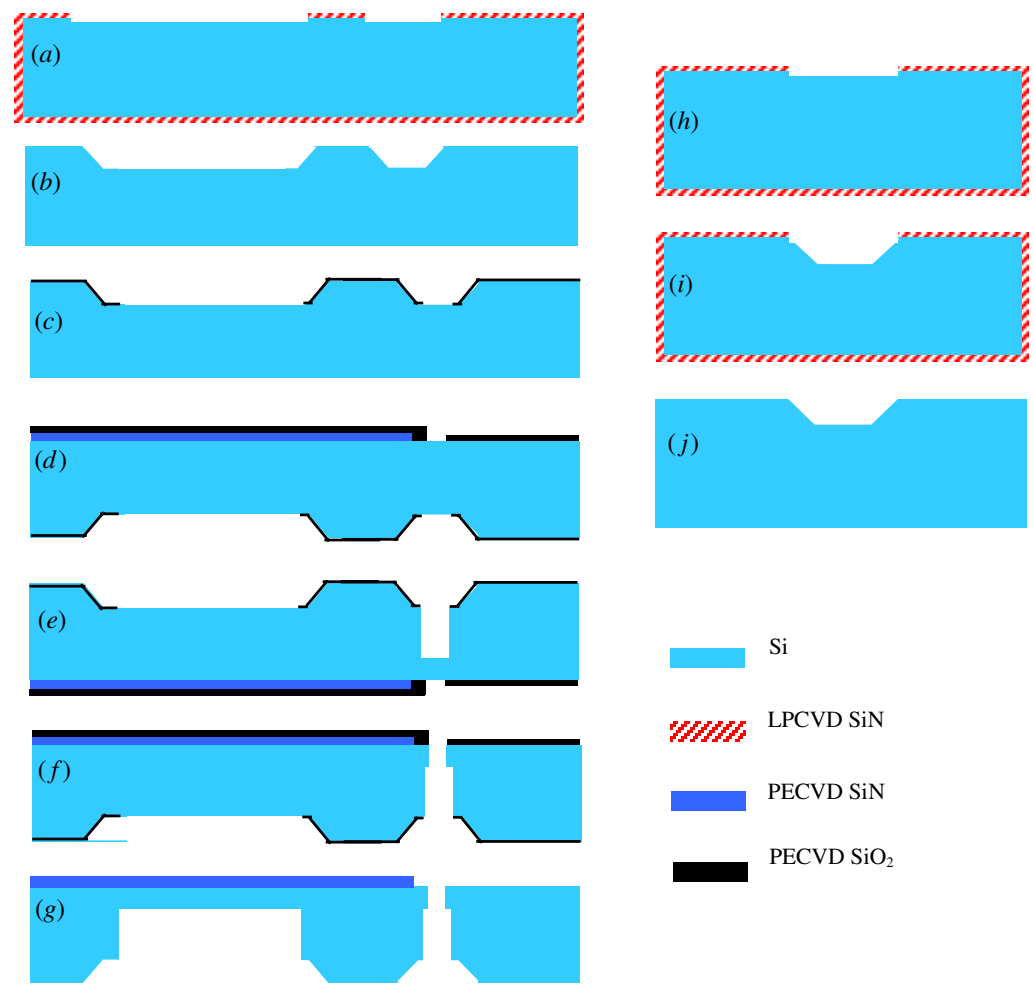

Figure 5. Schematic process flow for the cantilever-supported pyramid structures $(a)-(g)$ and V-pit structures $(h)-(j)$.

( $j$ ) Pyramids and pits with $100 \mu \mathrm{m}$ depth and smooth Si $\left\{\begin{array}{lll}1 & 1 & 1\end{array}\right\}$ sidewalls were achieved. The etch depth for the vernier scales was $2.8 \mu \mathrm{m}$ resulting from $4 \mu \mathrm{m}$ feature widths. Following the $\mathrm{SiN}$ mask strip, (c) $6 \mu \mathrm{m} \mathrm{SiO}_{2}$ was deposited by plasmaenhanced chemical vapour deposition (PECVD) on the front side ( $\mathrm{KOH}$ etch side) of the wafer containing pyramids to provide a conformal coverage on the pyramids. A $1 \mu \mathrm{m}$ thick aluminium (Al) layer was deposited by sputtering on top of the oxide layer for extra protection of the pyramids. A photoresist solution of AZ4562:MEK = 1:6 was then spray-coated on top of the $\mathrm{Al} / \mathrm{SiO}_{2}$ layers. After a hard bake, the patterned resist was used as a mask to wet etch the $\mathrm{Al}$ and $\mathrm{SiO}_{2}$ layers in a standard $\mathrm{Al}$ etchant and buffered HF (BHF) solutions, respectively. (d) On the backside of the wafer, a $3.5 \mu \mathrm{m}$ thick SiN layer with approximately $1 \mathrm{GPa}$ compressive stress was deposited by PECVD and patterned. Subsequently, $4 \mu \mathrm{m}$ thick PECVD $\mathrm{SiO}_{2}$ was deposited on top of the $\mathrm{SiN}$ and patterned with alignment to the $\mathrm{SiN}$ patterns. Sufficient margins between the formed pyramids and the supporting protrusions were included in the design and thus common optical alignment errors in a few micron ranges during $\mathrm{SiN}$ and $\mathrm{SiO}_{2}$ patterning could be tolerated. (e) Using the patterned oxide as the mask, deep reactive ion etching (DRIE) exploiting the Bosch process [15] was carried out from the wafer front achieving an etch depth of $300 \mu \mathrm{m}$ which defines the protrusion support for the pyramids and the cantilever thickness $(100 \mu \mathrm{m}) . \quad(f)$ The subsequent DRIE process on the wafer backside frees the cantilevers.

The wafers containing the fabricated micromechanical features were diced into $20 \mathrm{~mm} \times 20 \mathrm{~mm}$ chips followed by a short BHF dip to remove the oxide residue. Alignment and bonding were carried out immediately after the corresponding chips were cleaned in standard RCA1 $\left(\mathrm{H}_{2} \mathrm{O}_{2}: \mathrm{NH}_{4} \mathrm{OH}: \mathrm{H}_{2} \mathrm{O}=\right.$ 1:1:5) and $\mathrm{RCA} 2\left(\mathrm{H}_{2} \mathrm{O}_{2}: \mathrm{HCl}: \mathrm{H}_{2} \mathrm{O}=1: 1: 6\right)$ solutions, respectively. When stacking chip A on top of chip B as illustrated in figure 1 , the corners of the chips were initially used as a guide to align them coarsely. The engagement between the convex and concave features could then be indicated by the absence of relative movement between the chips when only the top chip A was pushed from a side of one of the corners. Followed by the engagement and alignment, local pressure was applied at the centre of the chip stack to initiate direct bonding. The bonded chips were then annealed at $400{ }^{\circ} \mathrm{C}$ in a $\mathrm{N}_{2}$ atmosphere for $2 \mathrm{~h}$. Both infrared (IR) and SEM have been used to inspect the bonding quality and the alignment accuracy.

\section{Results and discussion}

The alignment structures were successfully fabricated with the cantilever-supported pyramids shown in figure 6 . The sharp edges and smooth sidewalls of the pyramids are clearly observed which suggested effective CCCS for the $\mathrm{KOH}$ etching. Figure 7 shows the formed micro-vernier structures. The average dimensions of the fabricated pyramids, V-pits and vernier width are $358.5 \mu \mathrm{m}, 388 \mu \mathrm{m}$ and $4.7 \mu \mathrm{m}$, respectively, as measured using a high-resolution optical microscope. The slight over-etch was due to the slow $\mathrm{KOH}$ etch along the Si $\langle 111\rangle$ direction. The cantilever tip deflections are approximately $50-115 \mu \mathrm{m}$ depending on the lengths of the cantilevers. These deflections are sufficient for the engagement between the convex and concave features, as explained in section 2 . The cantilever displacements, however, are greater than the predicted values induced solely from 

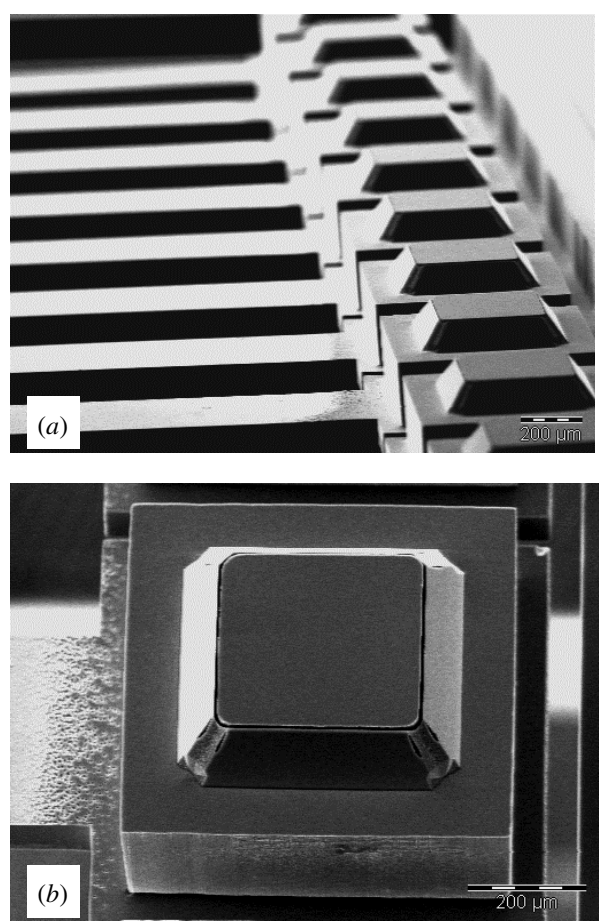

Figure 6. SEM micrographs of ( $a$ ) an array of cantilever-supported pyramids in a pitch and $(b)$ a typical convex pyramid structure.

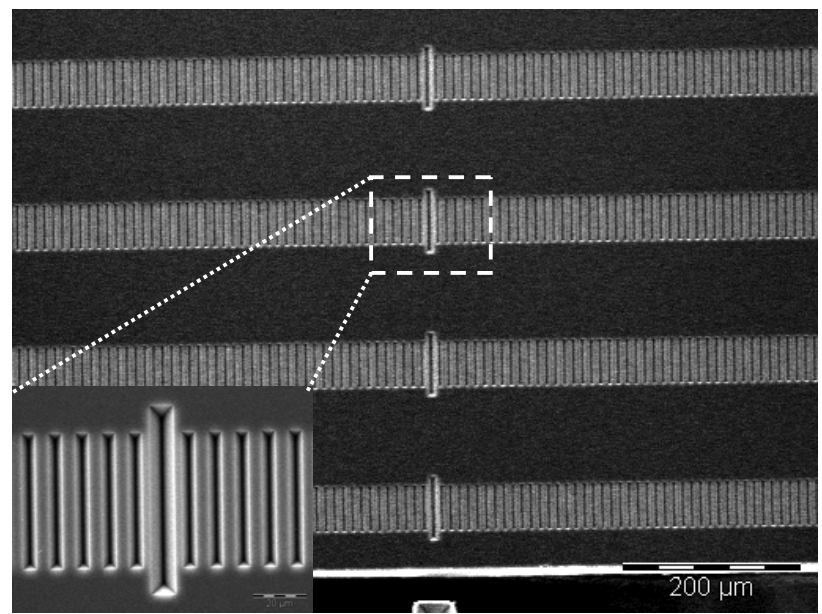

Figure 7. SEM image of typical micro-vernier rows with the inset showing the individual etched scales.

the SiN stress in figure 3. This implies that an additional compressive stress was generated during the microfabrication steps, which has also contributed to the cantilever bending. In order to examine the coupling at the bonding interface, a few of the cantilever features were broken off after the aligned bonding processes and figure 8 shows a tilted view of the aligned and bonded chip pair to reveal the engagement between the pyramid and the V-pit structures.

Infrared (IR) has been used to inspect the alignment accuracy by observing the overlapping micro-verniers at the bonding interface. Comparison with the designed verniers without misalignment in figure 4, a typical IR image in figure 9, suggests that the alignment is approximately $200 \mathrm{~nm}$ or better. To confirm the alignment accuracy, the bonded chip pairs were

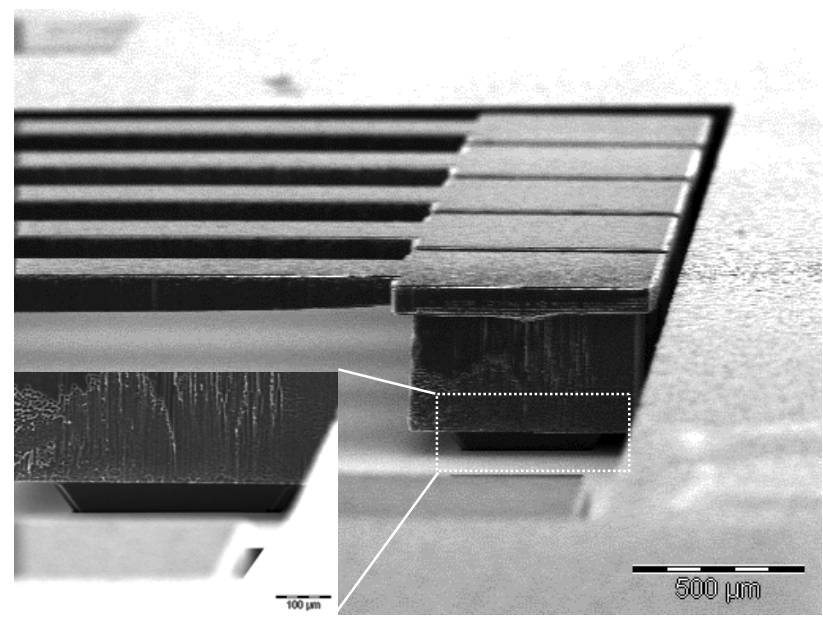

Figure 8. Tilted SEM image of a pair of chips after aligned bonding. The inset shows the engagement between the pyramids and pits.

diced through the overlapping vernier areas, as indicated in figure 4, and the diced profile has been inspected by SEM. The SEM images in figure 10 show both the middle and end regions of the overlapped verniers which confirm the alignment accuracy to be better than $200 \mathrm{~nm}$. Verniers in both $x$ and $y$ directions have been inspected and found to have the same alignment accuracy. This alignment precision has been achieved in approximately half of the bonded samples. We believe that the existence of the misalignment in some of the bonded samples could be attributed to the lack of specific control of the uniformity and directionality of the applied bonding pressure. The solutions to this could be incorporated in future work where uniform and controllable pressure could be implemented by using conventional chip/wafer bonders after the alignment step.

The bonding quality was also examined using IR inspection, as shown in figure 11. A successful aligned bonding is demonstrated to be free of voids in most of the bonding areas. The visible bonding defects in the corner area are probably due to the existence of the adjacent DRIE patterns as well as the non-uniformity of the applied pressure. The successful bonding result implies that the central bonding area was well protected during the microfabrication processes and thus good direct bonding was achieved. This was further confirmed by atomic force microscopy results, which indicated that the measured root-mean-square (RMS) roughness of the central area prior to bonding process is around 2-2.6 $\AA$ which is comparable to that of the starting Si wafers $(1.5-1.7 \AA)$. Thus, for future development, it would be safe to position MEMS/IC devices within this area for subsequent bonding processes.

The results suggest a practical and effective alignment technique that can be readily expanded to the wafer level. Electronic devices or micro/nano systems positioned in the central area of the wafers could be aligned and bonded with nanoprecision, thereby realizing multi-layered devices and nano-systems with advanced functionality such as for 3D IC integration. This alignment technique will be particularly suitable where the microfabrication processes for the alignment features are already required as steps in the fabrication for the devices to-be-bonded. Furthermore, 

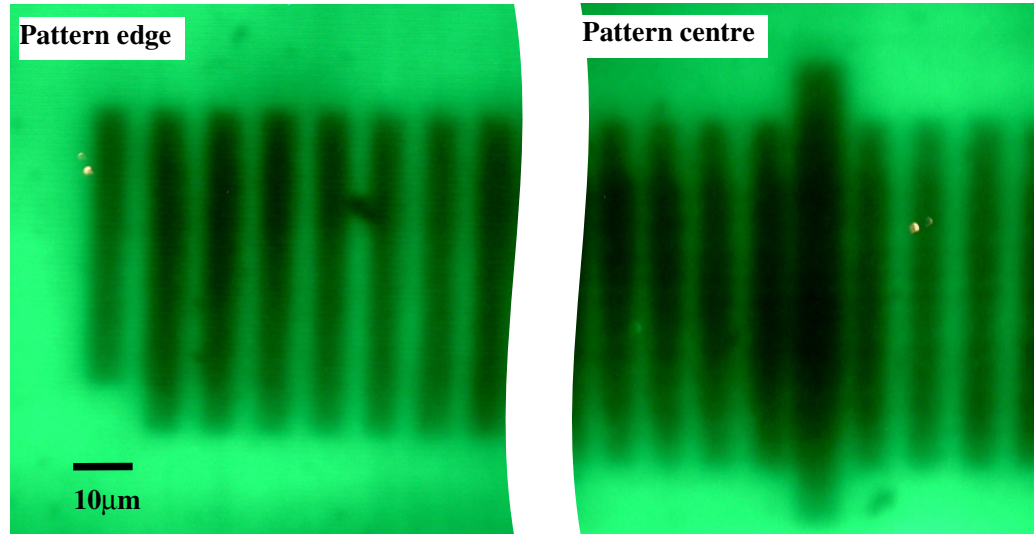

Figure 9. IR image from a pair of aligned and bonded chips to reveal the overlapping of the verniers.
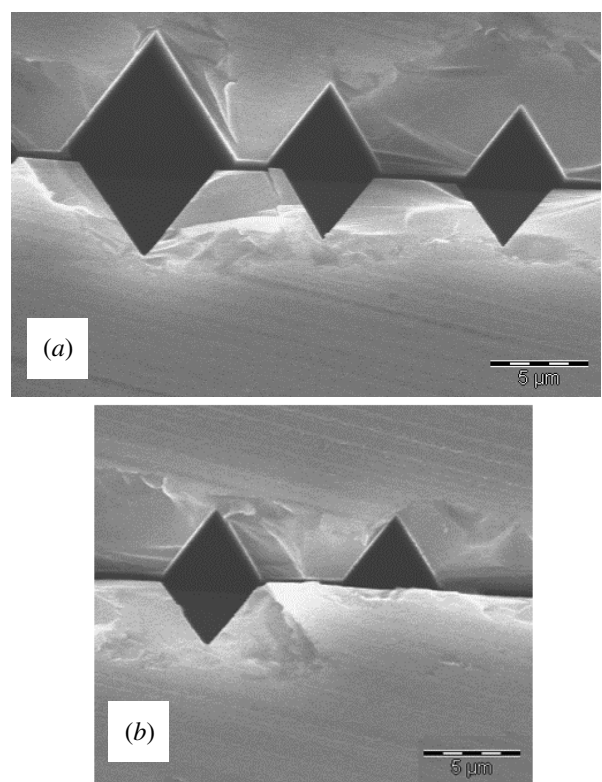

Figure 10. SEM profile image to reveal the aligned and bonded vernier scales in the $(a)$ middle area and $(b)$ end area of the verniers.

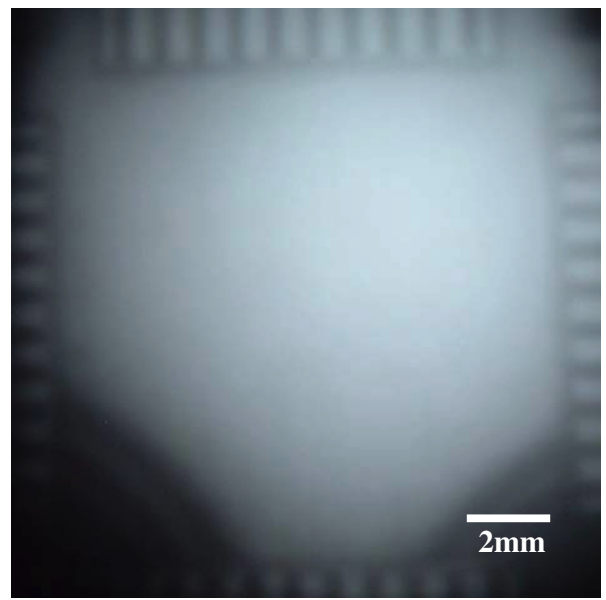

Figure 11. IR image from a bonded sample.

despite the fabrication processes required for the alignment features, this technique provides a viable approach in many applications where nanopresicion alignment is currently not possible or too costly to implement. For example, due to the novel micromechanical principles utilized in this technique, the developed approach could be particularly suitable for bonding with interlayer materials (glass, polymer and eutectic metals) which go through a softened or molten phase during the bonding processes, which makes the maintenance of alignment particularly problematic. In these cases, typical alignment accuracy is usually no better than a few microns [16]. The potential of the proposed bonding alignment technique could also be entailed in the assembly of optoelectronic microsystems which has hitherto been almost exclusively a manual and therefore expensive process. In particular, the demands for precision alignment are most pronounced in laser diodes with a maximum tolerance of $200 \mathrm{~nm}$ or less [17]. The technique described herein seems to be a promising solution to align and bond $\mathrm{Si}$ submounts accommodating optical fibres and laser devices leading to more cost-effective assembly.

\section{Conclusions}

A novel approach has been developed aiming at achieving nanoprecision bonding alignment using micromechanical structures. Convex and concave features comprised of pyramids and V-pits have been designed for passive bonding alignment at the $\mathrm{Si}$ chip level. The shape and position of the pyramids and V-pits provide kinematic averaging, while the compliance of the support cantilever structure introduces elastic averaging. Micro-vernier scales for subsequent bonding alignment inspection have also been designed at the bonding interface and fabricated together with the pyramids/pits as a one-mask process.

The alignment features were successfully fabricated and the engagement concept was implemented. Both IR and SEM profile images confirmed that an alignment accuracy of better than $200 \mathrm{~nm}$ was achieved at the bonding interface without the assistance of any optical alignment facilities. Our results suggest that the bonding interface is well protected during the entire microfabrication process and consequently good direct bonding was achieved as a result. This implies that future IC and micro/nano devices could be positioned within this central area for subsequent aligned bonding processes. Therefore, the developed technique provides a viable approach 
to improve significantly wafer bonding alignment thereby enable future high performance applications, particularly in micro-optoelectronic systems, 3D interconnect schemes and high performance MEMS.

\section{Acknowledgments}

We acknowledge the financial support from the University of Southampton Nanomaterials Forum. SMS holds a Royal Society-Wolfson Research Merit Award.

\section{References}

[1] Lasky J B 1986 Wafer bonding for silicon-on-insulator technologies Appl. Phys. Lett. 48 78-80

[2] Shimbo M, Furukawa K, Fukuda F and Tanzawa K 1986 Silicon-to-silicon direct bonding method J. Appl. Phys. 60 2987-9

[3] Miki N, Zhang X, Khanna R, Ayón A A, Ward D and Spearing S M 2003 Multi-stack silicon-direct wafer bonding for 3D MEMS manufacturing Sensors Actuators A 103 194-201

[4] Lu J Q et al 2002 A wafer-scale 3D IC technology platform using dielectric bonding glues and copper damascene patterned inter-wafer interconnects Proc. IEEE 2002 Int. Interconnect Technology Conf. pp 78-80

[5] Topol A W, Furman B K, Guarini K W, Shi L, Cohen G M and Walker G F 2004 Enabling technologies for wafer-level bonding of 3D MEMS and integrated circuit structures IEEE Proc. 54th Electronic Components and Technology Conf. pp 931-8

[6] Peizer R, Mafthias T, Kettner P, Lindner P and Schaefer C 2003 Vertical 3D interconnect through aligned wafer bonding Proc. 5th Int. Conf. on Electronic Packaging Technology vol 5 pp 512-7
[7] Gui C, Veldhuis G J, Koster T M, Lambeck P V, Berenschot J W, Gardeniers J G E and Elwenspoek M 1999 Fabrication of nanomechanical optical devices with aligned wafer bonding Microsyst. Technol. 5 138-43

[8] Mirza A R 2000 One micron precision, wafer-level aligned bonding for interconnect, MEMS and packaging applications IEEE Proc. 50th Electronic Components \& Technology Conf. pp 676-80

[9] Slocum A H and Weber A C 2003 Precision passive mechanical alignment of wafers IEEE J. Microelectromech. Syst. 12 826-34

[10] Seidel H, Csepregi L, Heuberger A and Baumgärtel H 1990 Anisotropic etching of crystalline silicon in alkaline solutions: I. Orientation dependence and behavior of passivation layers J. Electrochem. Soc. 137 3612-26

[11] Reddy P S and Jessing J R 2004 Pattern alignment effects in through-wafer bulk micromachining of $\left(\begin{array}{lll}1 & 0 & 0\end{array}\right)$ silicon Proc. 2004 IEEE Workshop on Microelectronics and Electron Devices pp 89-92

[12] Zhang Q, Liu L and Li Z 1996 A new approach to convex corner compensation for anisotropic etching of $\left(\begin{array}{lll}1 & 0 & 0\end{array}\right) \mathrm{Si}$ in KOH Sensors Actuators A 56 251-4

[13] Godin M, Tabard-Cossa V, Grutter P and Williams P 2001 Quantitative surface stress measurements using a microcantilever Appl. Phys. Lett. 79 551-3

[14] Stoney G 1909 The tension of metallic films deposited by electrolysis Proc. R. Soc. A 82 172-5

[15] Laermer F, Schilp A, Funk K and Offenberg M 1999 Bosch deep silicon etching: improving uniformity and etch rate for advanced MEMS applications IEEE Proc. 12th Int. Conf. on Micro Electro Mechanical Systems pp 211-6

[16] Niklaus F, Enoksson P, Kälvesten E and Stemme G 2003 A method to maintain wafer alignment precision during adhesive wafer bonding Sensors Actuators A 107 273-8

[17] Shakespeare W J 2005 MEMS integrated submount alignment for optoelectronics J. Lightwave Technol. 23 504-9 